Ferrata Storti Foundation

\title{
Disrupting the leukemia niche in the central nervous system attenuates leukemia chemoresistance
}

Haematologica 2020

Volume 105(8):2130-2140

\section{Correspondence: \\ PETER GORDON, \\ gord0047@umn.edu \\ Received: June 24, 2019. \\ Accepted: October 14, 2019. \\ Pre-published: October 17, 2019.}

doi:10.3324/haematol.2019.230334

Check the online version for the most updated information on this article, online supplements, and information on authorship \& disclosures: www.haematologica.org/content/105/8/2130

(C)2020 Ferrata Storti Foundation

Material published in Haematologica is covered by copyright. All rights are reserved to the Ferrata Storti Foundation. Use of published material is allowed under the following terms and conditions:

https://creativecommons.org/licenses/by-nc/4.0/legalcode. Copies of published material are allowed for personal or internal use. Sharing published material for non-commercial purposes is subject to the following conditions:

https://creativecommons. orǵ/licenses/by-nc/4.0/legalcode, sect. 3. Reproducing and sharing published material for commercial purposes is not allowed without permission in writing from the publisher.

\author{
Leslie M. Jonart, ${ }^{1,2}$ Maryam Ebadi, ${ }^{1,2}$ Patrick Basile, ${ }^{1,2}$ Kimberly Johnson, ${ }^{1,2}$ \\ Jessica Makori ${ }^{1,2}$ and Peter M. Gordon ${ }^{1,2}$
}

${ }^{1}$ Division of Pediatric Hematology and Oncology, Department of Pediatrics, University of Minnesota and ${ }^{2}$ Masonic Cancer Center, University of Minnesota, Minneapolis, MN, USA.

\section{ABSTRACT}

P rotection from acute lymphoblastic leukemia relapse in the central nervous system (CNS) is crucial to survival and quality of life for leukemia patients. Current CNS-directed therapies cause significant toxicities and are only partially effective. Moreover, the impact of the CNS microenvironment on leukemia biology is poorly understood. In this study we showed that leukemia cells associated with the meninges of xenotransplanted mice, or co-cultured with meningeal cells, exhibit enhanced chemoresistance due to effects on both apoptosis balance and quiescence. From a mechanistic standpoint, we found that leukemia chemoresistance is primarily mediated by direct leukemia-meningeal cell interactions and overcome by detaching the leukemia cells from the meninges. Next, we used a co-culture adhesion assay to identify drugs that disrupted leukemia-meningeal adhesion. In addition to identifying several drugs that inhibit canonical cell adhesion targets we found that Me6TREN (Tris[2-(dimethylamino)ethyl]amine), a novel hematopoietic stem cell-mobilizing compound, also disrupted leukemia-meningeal adhesion and enhanced the efficacy of cytarabine in treating CNS leukemia in xenotransplanted mice. This work demonstrates that the meninges exert a critical influence on leukemia chemoresistance, elucidates mechanisms of relapse beyond the well-described role of the bloodbrain barrier, and identifies novel therapeutic approaches for overcoming chemoresistance.

\section{Introduction}

Central nervous system (CNS) relapse is a common cause of treatment failure among patients with acute lymphoblastic leukemia (ALL). ${ }^{1.3}$ Relapses occur despite CNS-directed therapies which include high-dose systemic chemotherapy, intrathecal chemotherapy, and cranial irradiation in some high-risk patients. These current CNS-directed therapies are also associated with significant acute and long-term toxicities. ${ }^{4-10}$ Accordingly, novel CNS-directed leukemia therapies are needed to improve long-term outcomes in ALL while decreasing treatment-related morbidity.

Historically, the ability of leukemia cells and chemotherapy to access the restricted CNS environment has been posited as a critical factor in the pathophysiology of CNS leukemia and relapse. However, several lines of evidence suggest that this is an overly simplistic model. First, high rates $(>50 \%)$ of CNS leukemia occur in patients in the absence of adequate CNS-directed therapies as well as in mice transplanted with human, primary B-cell precursor leukemia cells. ${ }^{11-14}$ Moreover, clonal analyses of paired leukemia cells isolated from both the bone marrow and CNS of patients and xenotransplanted mice demonstrated that all, or most, B-cell ALL clones are capable of disseminating to the CNS..$^{14,15}$ Third, CNS leukemia relapses occur despite high-dose systemic and intrathecal chemotherapy. These therapies either overcome or bypass the blood-brain barrier. Fourth, it was shown that high Mer kinase-expressing, $t(1 ; 19)$ leukemia cells co-cultured with CNS-derived cells exhibit G0/G1 cell cycle arrest, suggestive of dormancy or quiescence, as well as 
methotrexate resistance. ${ }^{16}$ Similarly, Akers et al. showed that co-culture of leukemia cells with astrocytes, choroid plexus epithelial cells, or meningeal cells enhanced leukemia chemoresistance. ${ }^{17}$ Together these observations suggest that the pathophysiology of CNS leukemia extends beyond the role of the blood-brain barrier. We hypothesize that the ability of leukemia cells to persist in the unique CNS niche and escape the effects of chemotherapy and immune surveillance likely also play critical roles in CNS leukemia and relapse.

However, while extensive research has demonstrated a critical role of the bone marrow niche in leukemia biology, the impact of the CNS niche on leukemia biology is less well understood. ${ }^{18,19}$ Herein, we demonstrate that the meninges exert a unique and critical influence on leukemia biology by enhancing leukemia resistance to the chemotherapy agents currently used in the therapy of CNS leukemia. We then leveraged this new understanding of the mechanisms of meningeal-mediated leukemia chemoresistance to identify a novel drug, Me6TREN (Tris[2-(dimethylamino)ethyl]amine), which overcomes leukemia chemoresistance by disrupting the interaction between leukemia and meningeal cells.

\section{Methods}

\section{Cells and tissue culture}

Leukemia cells were obtained from the American Type Culture Collection (ATCC) or Deutsche Sammlung von Mikroorganismen und Zellkulturen (DSMZ) and cultured in RPMI medium (Sigma-Aldrich) supplemented with fetal bovine serum $10 \%$ (Seradigm) and penicillin-streptomycin (Sigma-Aldrich). Leukemia cell lines included both B-cell (NALM-6, SEM) and Tcell (Jurkat, SEM, MOLT-13) immunophenotypes. The HCN-2 neuronal cell line was obtained from the ATCC. Leukemia cells expressing green fluorescent protein (GFP) were generated as described elsewhere. ${ }^{20}$ Murine leukemia cells, generated by BCR/ABL p190 expression in hematopoietic cells from CD45.1 Arf $^{-/}$mice, ${ }^{21-23}$ were provided by Dr. Michael Farrar (University of Minnesota, MN, USA). Primary B-ALL cells for co-culture experiments were obtained from the University of Minnesota Hematologic Malignancy Bank (IRB \#: 0611M96846; pediatric patient at diagnosis). Primary B-ALL cells for in vivo experiments were obtained from the Public Repository of Xenografts [PRoXe; ${ }^{24}$ sample CBAB-62871-V1; pediatric patient at diagnosis with a $\mathrm{t}(4 ; 11)$ translocation]. Primary meningeal cells were obtained from ScienCell and cultured in meningeal medium supplemented with fetal bovine serum $2 \%$, growth supplement, and penicillin-streptomycin. Meningeal cells were isolated from multiple different donor specimens and were typically used between passages 3-5.

\section{Murine experiments}

NSG (NOD.Cg-Prkdcscid, Il2rgtm1 Wjl/SzJ; Jackson Laboratory) mice were housed under aseptic conditions. Mouse care and experiments were in accordance with a protocol approved by the Institutional Animal Care and Use Committee at the University of Minnesota (IRB\#1704-34717A). Mice 6-8 weeks old were injected intravenously via the tail vein with $\sim 1-2 \times 10^{6}$ human leukemia cells. Experiments were then performed 3-5 weeks after injection. In general, after euthanasia of the mice, the heart was perfused with phosphate-buffered saline and the meninges or other tissues were removed using a dissecting microscope, and dissociated by gently washing through a 0.40 $\mu \mathrm{m}$ filter (Millipore). Cells were then stained with fluorescent antibodies against CD19 (NALM-6; eBioscience) or CD3 (Jurkat, eBioscience) and assessed for apoptosis or cell cycle as described. Alternatively, leukemia cells could be purified from meningeal cells using immunomagnetic separation and either CD19 or CD3 antibodies (Stem Cell Technologies or Miltenyi Biotec) and placed back into suspension. In drug treatment experiments, Me6TREN $10 \mathrm{mg} / \mathrm{kg}$ and cytarabine $50 \mathrm{mg} / \mathrm{kg}$ were given by subcutaneous and intraperitoneal injection, respectively. Cerebrospinal fluid was obtained from mice as described elsewhere..$^{25}$ Briefly, mice were euthanized and a scalp incision was made at the midline to expose the dura mater overlying the cisterna magna. Under a dissection microscope, a tapered, pulled glass capillary tube was then inserted through the dura and into the cisterna magna to obtain clear cerebrospinal fluid. Experiments with murine leukemia cells (BCR/ABL p190 expression in hematopoietic cells from Arf $^{-1}$ mice; CD 45.1 background) used C57BL/6 mice. In these experiments, 3000 leukemia cells/mouse were injected via the tail vein.

\section{Statistical analysis}

Results are shown as the mean \pm standard error of mean of the results of at least three experiments. The Student $t$-test or analysis of variance was used for statistical comparisons between groups. The log-rank (Mantel-Cox) test was used to calculate $P$ values comparing the mouse survival curves. $P$ values $<0.05$ were considered statistically significant. Statistical analyses were conducted using GraphPad Prism 7 software (GraphPad Software, La Jolla, CA, USA).

\section{Results}

\section{Leukemia cells reside in the meninges of the mouse central nervous system}

In order to identify the anatomic site(s) in the CNS within which the leukemia cells reside, we transplanted multiple human ALL cell lines, including NALM-6, Jurkat, and SEM, into immune-compromised mice (NSG) via tail vein injection (Online Supplementary Figure S1A). Mice were not irradiated or conditioned with busulfan prior to transplantation to avoid perturbing leukemia niches. The mice were then euthanized and the CNS examined by histopathology and immunohistochemistry. We identified both the meninges and, to a lesser extent, the choroid plexus as the predominant CNS sites that harbor leukemia cells both before and after treatment with systemic cytarabine (Online Supplementary Figure S1B). In contrast, parenchymal involvement by leukemia was a rare, and often late, finding. It is possible that the altered immune system of NSG mice could influence CNS leukemia involvement or anatomic distribution. Accordingly, we also tested a pre-B-ALL mouse leukemia model that utilizes BCR/ABL p190 expression in hematopoietic cells from $A r f^{\prime}$ mice transplanted into immunocompetent mice. ${ }^{21,22,26}$ Similar to the xenotransplantation results, leukemia extensively involved the meninges in these mice (Online Supplementary Figure S1C).

\section{The meninges enhance leukemia chemoresistance}

We then developed ex vivo co-culture approaches to focus more specifically on the effects of the meninges on leukemia chemosensitivity. We selected meningeal cells based on our immunohistochemical analyses of brains 
from transplanted mice (Online Supplementary Figure $S 1 B$, $C)$ as well as histopathological examinations of brains from patients with leukemia. ${ }^{11.14} \mathrm{We}$ found that leukemia cells adhered to primary human meningeal cells in a co-culture system (Figure 1A). Moreover, leukemia cells co-cultured with meningeal cells were significantly more resistant to cytarabine and methotrexate-induced apoptosis, as measured by annexin- $\mathrm{V}$ and viability staining, relative to the same cells grown in suspension or adherent to the HCN-2 neural precursor cell line (Figure $1 \mathrm{~B}$ and Online Supplementary Table S1). In these experiments, chemotherapy had only very modest effects on meningeal cell viabil- ity (Online Supplementary Figure S2). Additionally, primary pre-B leukemia cell survival both in the presence and absence of chemotherapy was also enhanced when the cells were co-cultured with primary meningeal cells (Figure 1C). These results show that meningeal cells protect leukemia cells from the effects of cytotoxic chemotherapy.

We then used conditioned media from primary human meningeal cells to test whether direct cell-cell contact is required for meningeal-mediated leukemia chemoresistance. As shown in Figure 1D, meningeal conditioned media conferred moderate chemoresistance on leukemia cells, but to a lesser extent than co-culture. Together these
A

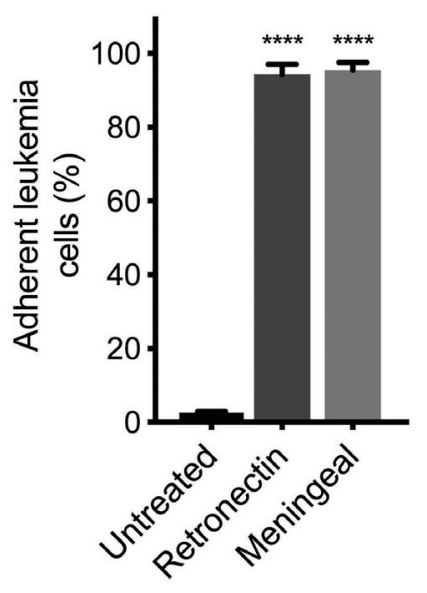

C

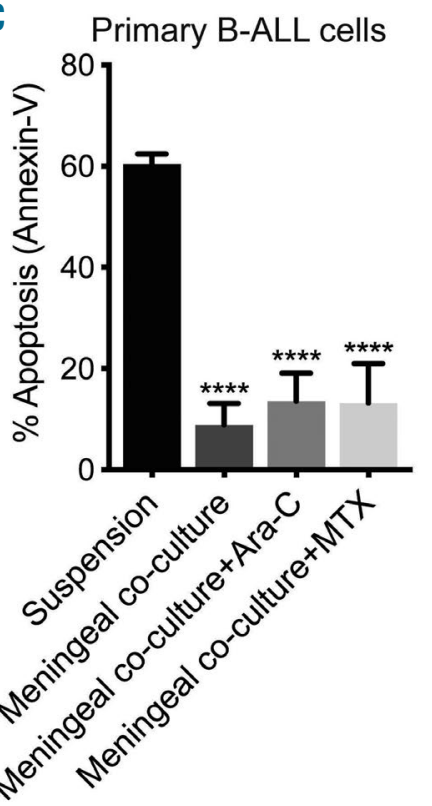

B

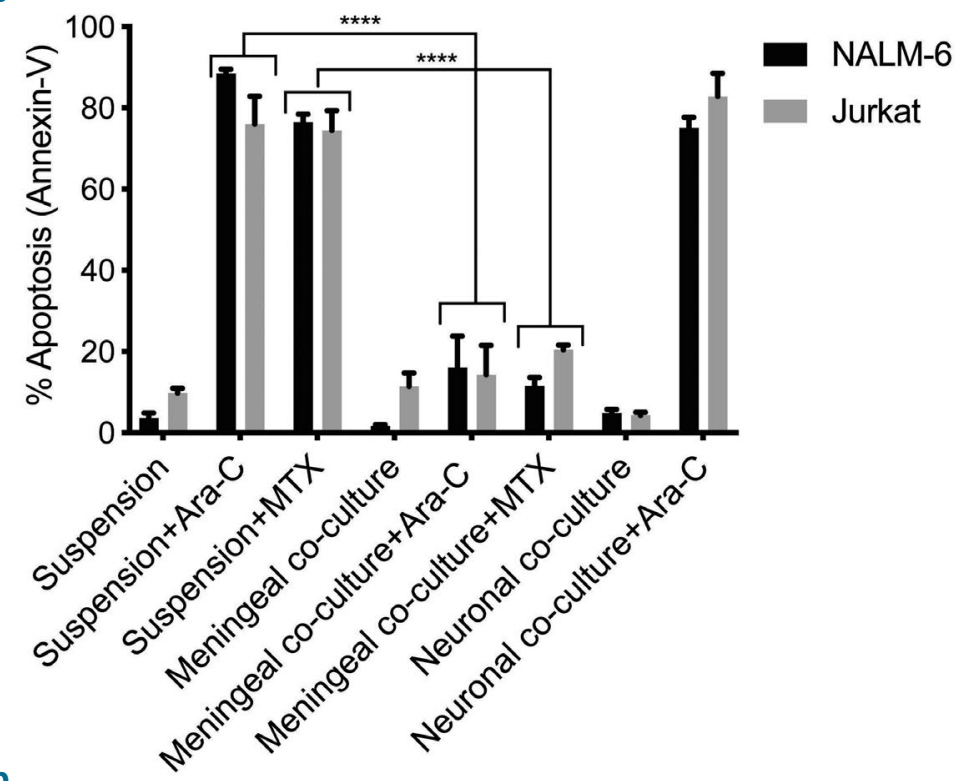

D

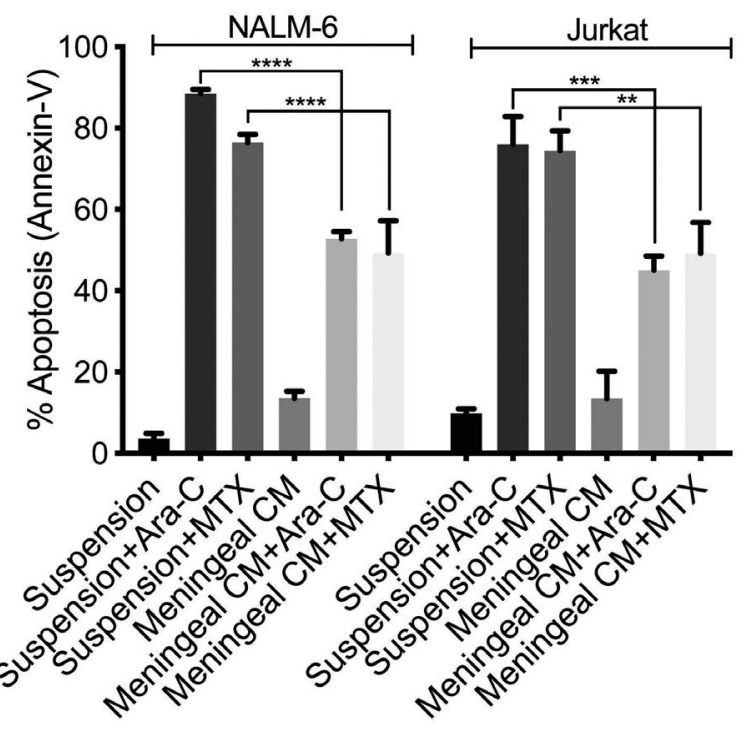

Figure 1. Leukemia cells exhibit increased chemoresistance when co-cultured with meningeal cells. (A) Percent of NALM-6 leukemia cells adherent to primary human meningeal cells, retronectin (recombinant fibronectin fragment) positive control, or non-tissue culture treated well after 2 h. (B, C) NALM- 6 and Jurkat leukemia cells (B) or primary B-cell acute lymphocytic leukemia cells (C) cultured in suspension or adherent to central nervous system-derived cells (primary human meningeal cells or the HCN-2 neuronal cell line) were treated with cytarabine $500 \mathrm{nM}$ or methotrexate $500 \mathrm{nM}$ for $48 \mathrm{~h}$ and then apoptosis was measured using annexin-V and viability staining and flow cytometry. (D) NALM-6 and Jurkat cells were cultured in either regular media or meningeal conditioned media (CM; $100 \%)$ and treated with cytarabine $500 \mathrm{nM}$ or methotrexate $500 \mathrm{nM}$ for $48 \mathrm{~h}$ before apoptosis was measured using annexin-V staining and flow cytometry. For all graphs, data are the mean \pm standard error of mean from three independent experiments. $* * P<0.01, * * * P<0.001, * * * * P<0.0001$ by analysis of variance. 
data suggest that meningeal-mediated leukemia chemoresistance is primarily dependent upon cell-cell contact with smaller contributions from a soluble factor(s) secreted by meningeal cells.

\section{Meningeal cells shift the apoptotic balance toward survival in leukemia cells}

In order to define the mechanism of meningeal-mediated leukemia chemoresistance we next examined the effects of co-culture on the apoptosis pathway in leukemia cells. In agreement with the annexin- $V$ results (Figure 1B), co-culture of leukemia cells with primary meningeal cells attenuated apoptosis caused by cytarabine or methotrexate treatment as determined by both caspase $3 / 7$ activity and measurement of leukemia cell mitochondrial potential with the dye TMRE (Figure 2A, B and Online Supplementary Figure S3). Next, we used an apoptosis antibody array to identify changes in the expression of multiple apoptosis family proteins in leukemia cells cocultured with meningeal cells relative to suspension. In particular, the levels of several pro-apoptotic proteins (BID, caspase $3 \& 8$ ) decreased in leukemia cells co-cultured with meningeal cells (Figure 2C). However, assessing how the dynamic levels, activities, and complex interactions of these and other BCL-2 family of pro-and antiapoptotic proteins (BH3 proteins) integrate to regulate the overall apoptotic balance is experimentally challenging. To try and capture the overall effect of the meninges on leukemia apoptotic balance, we then utilized $\mathrm{BH} 3$ profiling, a functional apoptosis assay that uses the response of mitochondria to perturbations by $\mathrm{BH} 3$ domain peptides, such as BIM, to predict the degree to which cells are primed to undergo apoptosis by the mitochondrial pathway. ${ }^{27,28}$ Importantly, decreased mitochondrial priming following drug treatment has been shown to be highly predictive of chemotherapy resistance in vitro and in vivo. ${ }^{27,28}$ Accordingly, we found that leukemia cells co-cultured with meningeal cells exhibited increased cytochrome C retention upon exposure to the BIM peptide compared to leukemia cells in suspension (Figure 2D). These data suggest that leukemia cells co-cultured with meningeal cells are significantly less primed to undergo apoptosis through the mitochondrial pathway than the same leukemia cells in suspension.

\section{Meningeal cells increase leukemia quiescence}

We also examined the effect of the meninges on leukemia cell cycle and quiescence. As shown in Figure 3A-C, leukemia cells co-cultured with primary meningeal cells are less proliferative, as determined by decreased Ki67 staining, significantly decreased S phase, and increased G0/G1 phase. We next used Hoechst-pyronin Y staining to better distinguish between G0 and G1 phases. ${ }^{29}$ As shown in Figure 3D-F, leukemia cells in co-culture with primary meningeal cells exhibited increased G0 phase, indicative of quiescence, relative to leukemia cells in suspension. Glucose uptake also diminished in leukemia cells co-cultured with meningeal cells, further supporting that these leukemia cells are quiescent (Online Supplementary Figure S4).

We then examined cell cycle and quiescence in leukemia cells isolated from the meninges of xenotransplanted mice. As shown in Figure 4A-C, leukemia cells isolated from the meninges of transplanted mice exhibited increased G0/G1 phase and decreased Ki-67 staining rela- tive to leukemia cells isolated from peripheral blood or bone marrow. In order to assess whether the meninges harbor long-term quiescent leukemia cells, we labeled leukemia cells with a fluorescent membrane dye (DiR or $\mathrm{DiD})$ that is retained in dormant, non-cycling cells but is diluted to undetectable levels within three to five generations in proliferating leukemia cells. ${ }^{30-34}$ Dye-labeled leukemia cells were then transplanted into immunodeficient mice. After having developed systemic leukemia in $\sim 4$ weeks, the mice were euthanized and their meninges harvested. Within the meninges we detected low levels of dye-retaining, quiescent leukemia cells by flow cytometry ( $<1 \%$ of total leukemia population) (Figure 4D, E). Further supporting that these dye-retaining leukemia cells are quiescent, the dye-retaining leukemia cells from the meninges exhibited low expression of the proliferation marker Ki-67 (Figure 4F). We also detected these quiescent leukemia cells within the meninges using confocal microscopy (Figure 4G-I).

We next treated these xenotransplanted mice with cytarabine and measured the percentage of dye-retaining leukemia cells in the meninges by flow cytometry. Cytarabine was given at a dose previously shown to result in plasma levels in the range produced by human highdose cytarabine regimens that cross the blood-brain barrier. ${ }^{35,36}$ Cytarabine, compared to phosphate-buffered saline, also significantly reduced the CNS leukemia burden in xenotransplanted mice, providing functional data that cytarabine is crossing the blood-brain barrier in our murine experiments (Online Supplementary Figure S5). Moreover, the relative increase in dye-retaining leukemia cells after cytarabine treatment is consistent with these cells having increased chemoresistance compared to the dye-negative, proliferating leukemia cells (Figure 4J). Together these data suggest that the meninges harbor quiescent leukemia cells that are resistant to chemotherapy.

\section{Meningeal-mediated leukemia chemoresistance is a reversible phenotype}

We then tested whether removal of leukemia cells from co-culture with meningeal cells restored chemosensitivity. Leukemia cells were dissociated from meningeal cells, purified with CD19 (NALM-6) or CD3 (Jurkat) magnetic beads, and placed back in suspension. As shown in Figure $5 \mathrm{~A}$, leukemia cells removed from co-culture exhibited similar sensitivity to methotrexate and cytarabine as leukemia cells in suspension. Likewise, leukemia cells grown in suspension after isolation from the meninges of xenotransplanted mice also exhibited sensitivity to cytarabine (Figure 5B). Further supporting these results, the cell cycle and apoptosis characteristics of leukemia cells placed back into suspension after co-culture reverted back to baseline (Figure $5 \mathrm{C}-\mathrm{E}$ ). These results suggest that drugs that disrupt adhesion between leukemia and meningeal cells may restore leukemia chemosensitivity in the CNS niche.

\section{Overcoming meningeal-mediated leukemia chemoresistance by disrupting adhesion}

Given that meningeal-mediated leukemia chemoresistance is a reversible phenotype, we next identified several cell adhesion inhibitors that disrupted leukemiameningeal adhesion in co-culture (Online Supplementary Figure S6A). We selected Me6TREN for further testing based upon its effectiveness in co-culture, small molecular 
weight (increasing its likelihood of CNS penetration), tolerability in mice, lack of prior testing in leukemia, and likely multifactorial mechanism of action. ${ }^{37,38}$ Me6TREN significantly disrupted the adhesion of both NALM- 6 and Jurkat leukemia cells to primary meningeal cells in a dosedependent fashion (Figure 6A and Online Supplementary Figure $S 6 B, C)$. This effect of Me6TREN on adhesion was not due to toxicity to leukemia or meningeal cells (Online Supplementary Figure S6D). We then quantified non-adherent leukemia cells in the cerebrospinal fluid of xenotransplanted mice after treatment with either Me6TREN or phosphate-buffered saline control in order to measure dis- ruption of leukemia adhesion in vivo. As shown in Figure 6B, Me6TREN-treated mice showed a modest, but significant, increase in leukemia cells in the cerebrospinal fluid, consistent with the co-culture experiment. Moreover, by disrupting leukemia adhesion, Me6TREN significantly attenuated leukemia chemoresistance in co-culture with meningeal cells (Figure 6C). We next assessed the in vivo ability of Me6TREN to enhance the efficacy of cytarabine in treating leukemia in the meninges. We tested NALM-6, Jurkat, and primary B-ALL leukemia cells with dosing regimens shown in Online Supplementary Figure S7. In all cases, Me6TREN significantly enhanced the efficacy of
A

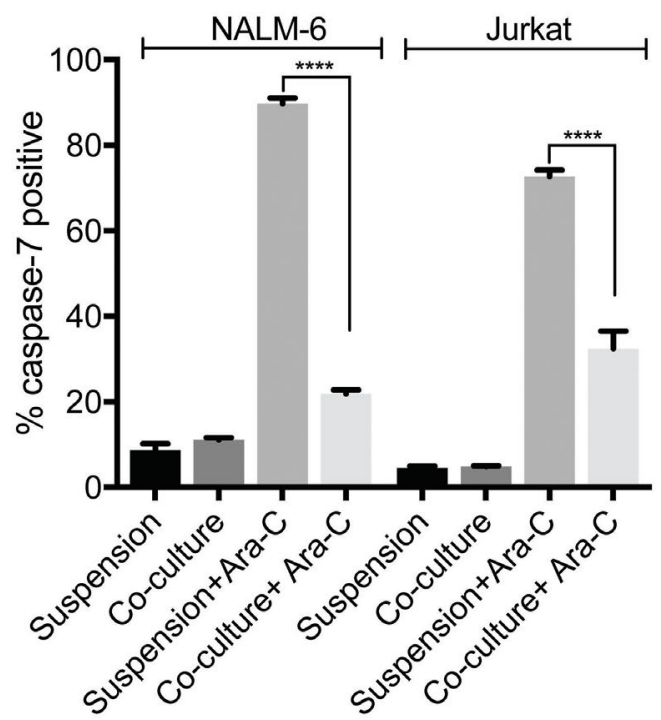

C

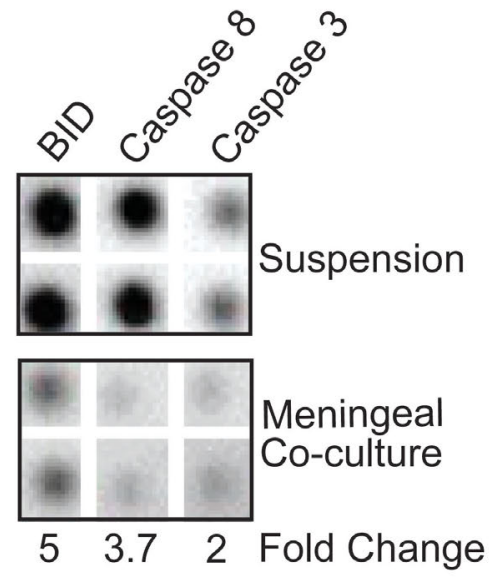

B

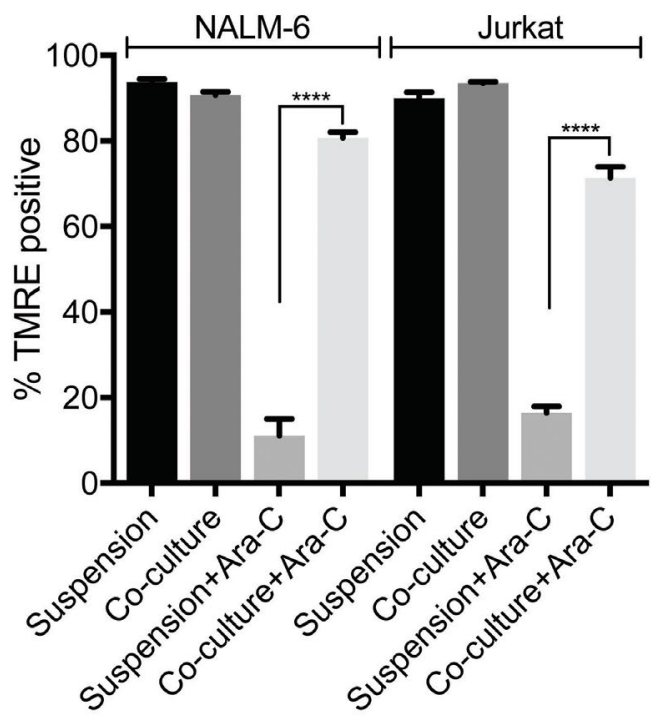

D

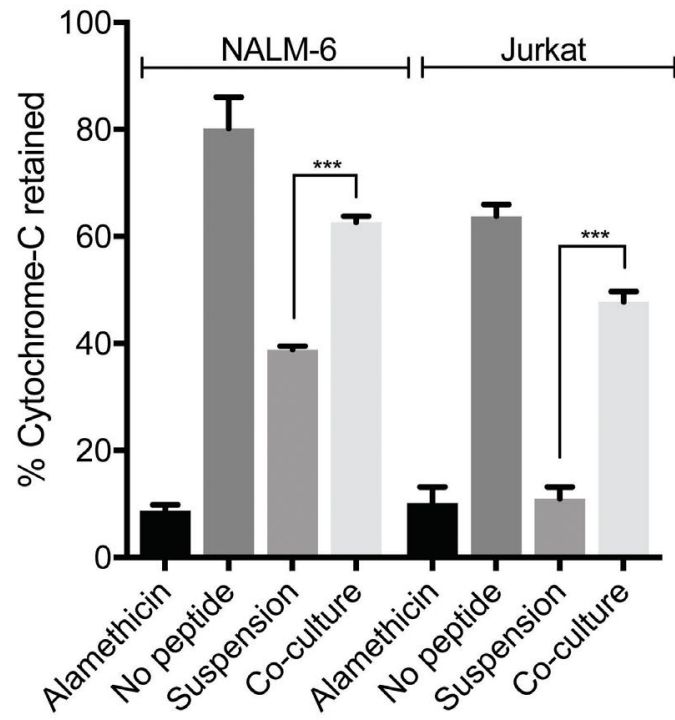

Figure 2. Meningeal cells tilt the apoptotic balance of leukemia cells toward survival. (A, B) NALM-6 and Jurkat leukemia cells cultured in suspension or adherent to meningeal cells were treated with cytarabine $500 \mathrm{nM}$ for $48 \mathrm{~h}$ and caspase-7 activity (A) and TMRE staining (B) assessed by flow cytometry. (C) NALM- 6 leukemia cells were grown in suspension or adherent to meningeal cells for $48 \mathrm{~h}$. Acute lymphocytic leukemia cells were then isolated and lysed. Protein lysate was used to probe a Human Apoptosis Antibody Array (Abcam). Representative portions of the membrane are shown. Relative protein expression was calculated after normalization using the IgG positive control. (D) BH3 profiling was performed using the BIM peptide on NALM- 6 and Jurkat leukemia cells grown in suspension or adherent to meningeal cells for $24 \mathrm{~h}$ (NALM-6) or $48 \mathrm{~h}$ (Jurkat). Cytochrome c retention was measured by flow cytometry. Alamethicin is a peptide antibiotic that permeabilizes the mitochondrial membrane and serves as a positive control. For all graphs, data are the mean \pm standard error of mean from three independent experiments. $* * P<0.01, * * * P<0.001, * * * * P<0.0001$ by analysis of variance. 
cytarabine in reducing the number of viable leukemia cells in the meninges (Figure 6D-G). Moreover, Me6TREN significantly extended the survival of mice treated with cytarabine in the patient-derived xenotransplantation model (Online Supplementary Figure S8). Despite Me6TREN disrupting the bone marrow hematopoietic niche, ${ }^{38}$ mice receiving cytarabine/Me6TREN or cytarabine alone exhibited comparable hematologic toxicities (Online Supplementary Figure S9). Finally, disruption of the CNS leukemia niche with Me6TREN did not result in an increased leukemia burden in other organs or tissues (Online Supplementary Figure S10).

We then performed gene expression profiling on primary, human meningeal cells treated with Me6TREN in order to take an unbiased approach toward identifying the mechanisms by which Me6TREN disrupts leukemiameningeal adhesion. As predicted, pathway analyses identified cell adhesion and migration as being among the most differentially regulated in meningeal cells treated with Me6TREN (Online Supplementary Figure S11A and Online Supplementary Table S2). The gene expression data also showed that Me6TREN significantly downregulated the cell-surface/adhesion proteins VCAM-1 and CD99 (Online Supplementary Figure S11B). Supporting a functional role for CD99 and VCAM-1 in leukemia-meningeal adhesion we found that antibodies targeting either of these proteins attenuated the adhesion of leukemia and meningeal cells (Online Supplementary Figure S11C, D). We also examined matrix metalloproteases (MMP) because Me6TREN has been shown to upregulate MMP-9 in the context of hematopoietic stem cells and the bone marrow niche..$^{38}$ Supporting a similar role in the CNS niche, two different MMP inhibitors diminished the ability of Me6TREN to disrupt leukemia-meningeal adhesion (Online Supplementary Figure S11C, D). In contrast, Me6TREN did not significantly perturb the CXCR4 and CCR7 signaling pathways, which have been previously implicated in leukemia infiltration into the CNS (Online Supplementary Figure S12 and Online Supplementary Table S3). ${ }^{39,40}$

\section{Discussion}

Protection from leukemia relapse in the CNS is crucial to long-term survival and quality of life of patients with leukemia. ${ }^{1-3}$ One strategy for developing novel CNSdirected therapies has focused on identifying, and potentially targeting, the factors that facilitate leukemia cell trafficking to the CNS from the bone marrow. ${ }^{39,41-49}$ At the
A

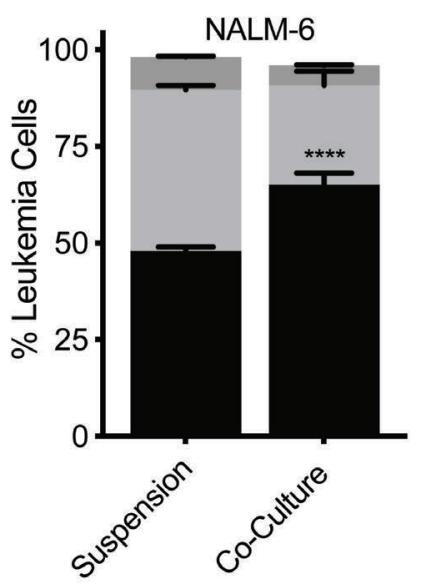

D

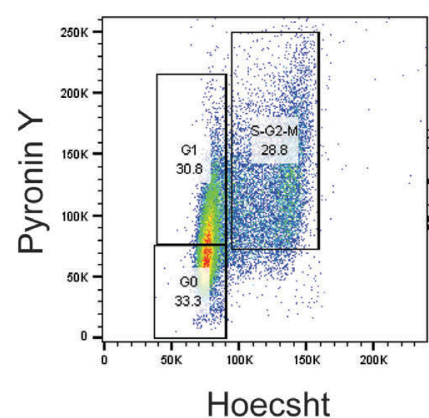

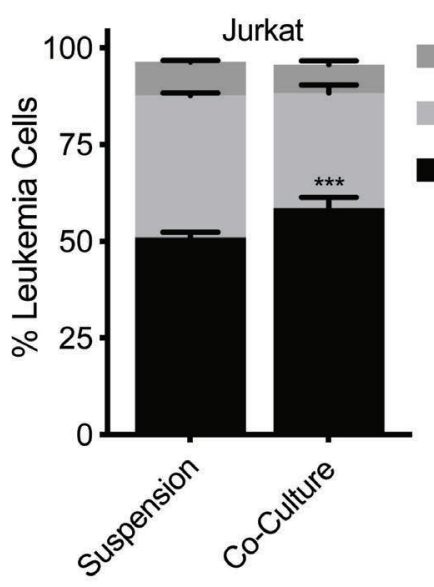

E

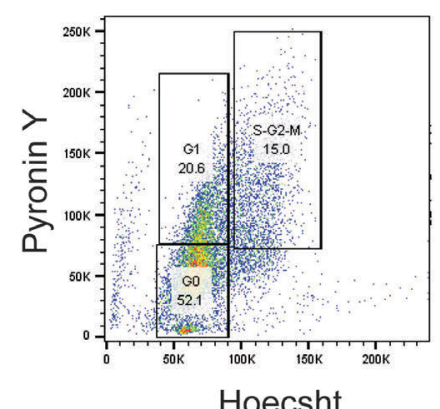

C

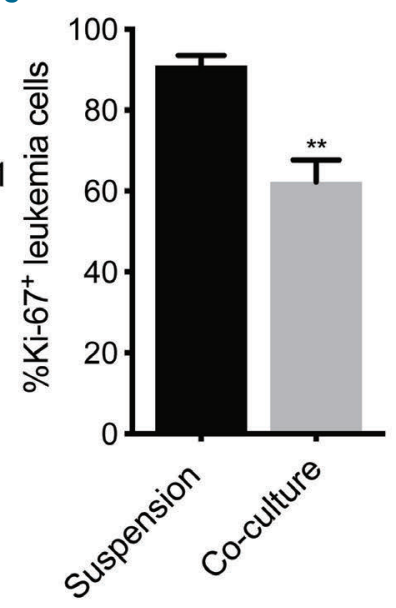

$\mathrm{F}$

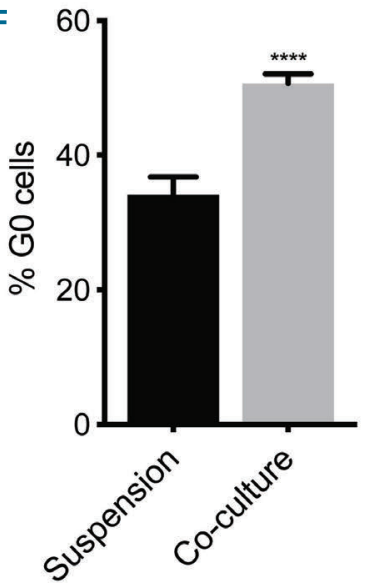

Figure 3. Meningeal cells increase leukemia quiescence in vitro. (A, B) NALM-6 and Jurkat leukemia cells cultured in suspension or adherent to primary meningeal cells for $48 \mathrm{~h}$ were assessed for cell cycle and proliferation using a Click-iT Plus EdU kit and flow cytometry. (C-F) NALM-6 leukemia cells cultured in suspension or adherent to primary meningeal cells for $48 \mathrm{~h}$ were stained for Ki-67 (C) or Hoechst-pyronin Y (D-F) and analyzed by flow cytometry. Representative flow plots for Hoechst-pyronin $Y$ staining are shown (D-E). For all graphs, $* P<0.05, * * P<0.01, * * * P<0.001, * * * * P<0.0001$ by analysis of variance. 
same time, additional evidence suggests that the ability of leukemia cells to infiltrate the CNS is a general property of most leukemia cells and not restricted to rare clones that acquire a metastatic phenotype. ${ }^{14,15}$ Accordingly, we sought to address the question of how leukemia cells adapt to this unique niche and escape the effects of chemotherapy after infiltrating the CNS. We found that, within the CNS, leukemia cells primarily localize to the meninges and that parenchymal involvement by leukemia was a rare finding. This observation is in agreement with a larger body of literature demonstrating that leukemia xenografts accurately model the anatomic distribution of leukemia observed within the CNS of humans. ${ }^{11-14}$ As a result, we focused our work on the meninges. However, it is certainly possible, and perhaps even likely, that other cells or tissues within the CNS, such as the choroid plexus, may also affect leukemia biology. ${ }^{17,20}$ This may be analogous to the bone marrow microenvironment in which distinct niches (endosteal, vascular) exert unique effects on hematopoietic stem and leukemia cells. ${ }^{50}$

We then used co-culture and in vivo xenotransplantation approaches to further characterize the effects of the
A

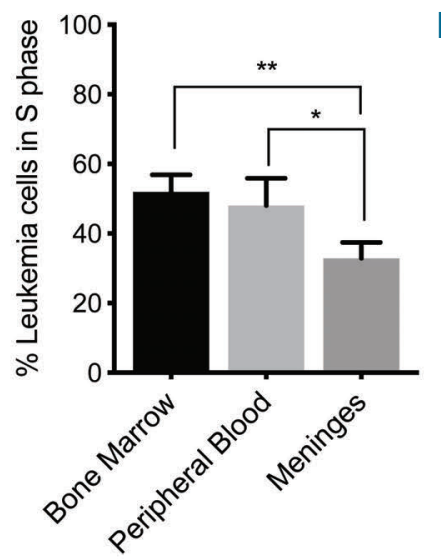

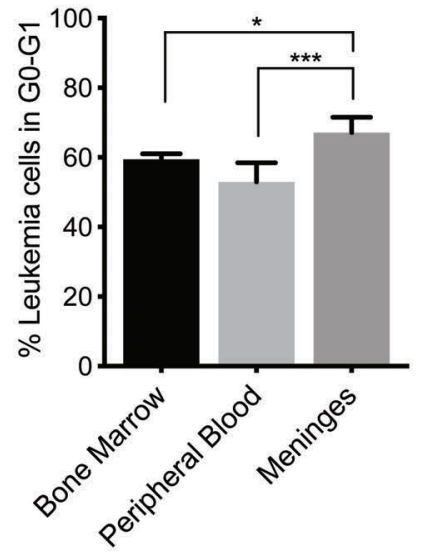

E

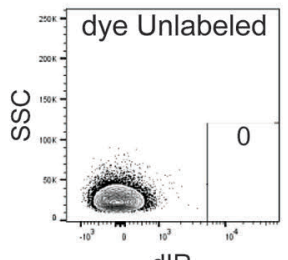

dIR

G

H
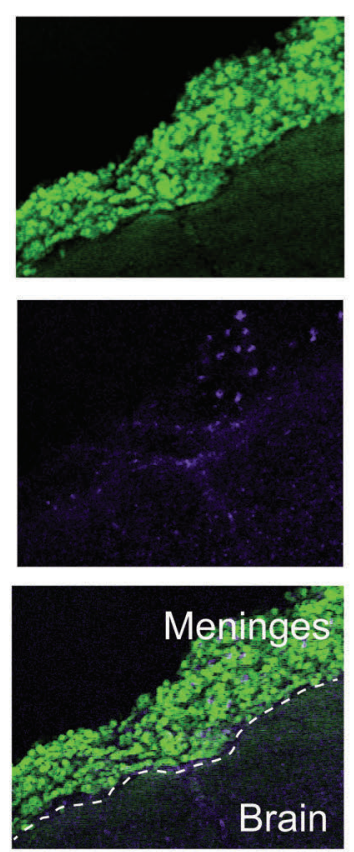

$J$

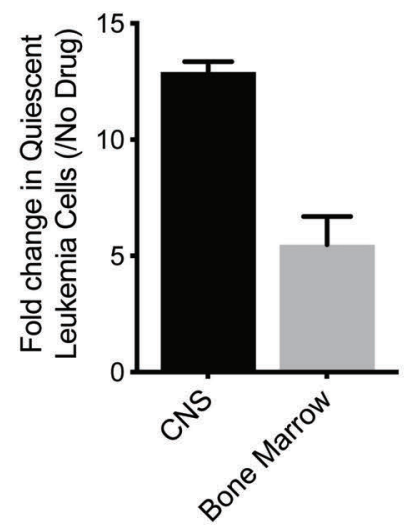

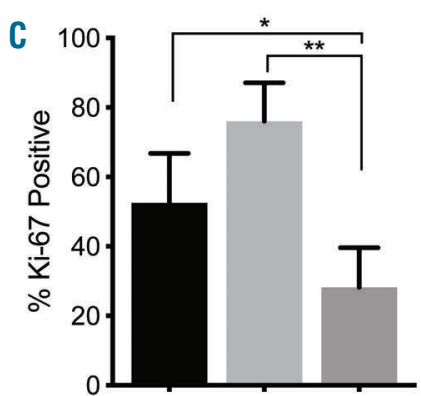
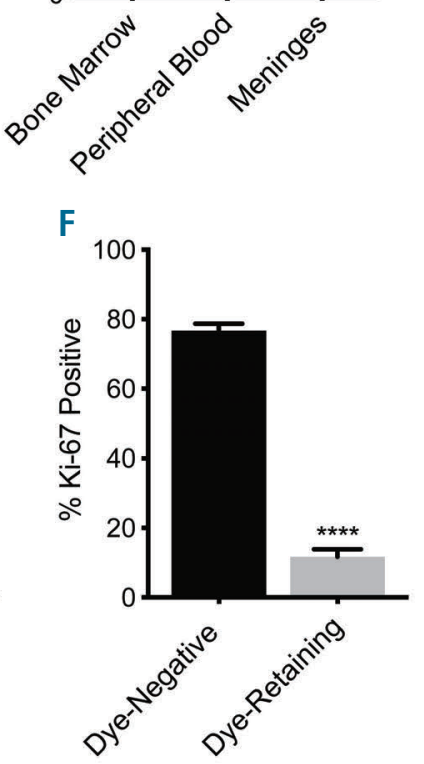

Figure 4. The meninges harbor quiescent and chemoresistant leukemia cells in vivo. (A-C) Mice were transplanted with NALM-6 leukemia cells ( $2 \times 10^{6}$ cells; $n=5$ per group). After 4 weeks, mice were injected intravenously with Click-iT EdU cell cycle reagent prior to harvesting tissues (meninges, bone marrow, and peripheral blood) and assessing for cell cycle (A-B) and the proliferation marker Ki-67 (C) by flow cytometry. (D-F) Mice were transplanted with membrane dye-labeled (DiD) or control, unlabeled NALM- 6 leukemia cells $\left(2 \times 10^{6}\right.$ cells) and then euthanized 4 weeks later $(n=5)$. Ki-67 negative, quiescent (dye-positive) acute lymphoblastic leukemia (ALL) cells were identified and quantified (D-F) in the meninges by flow cytometry. Representative flow cytometry plots are shown in (D). (G-I) Confocal microscopy images showing total (G; green), dye-retaining, quiescent $(\mathrm{H}$; purple), and overlay (I) of leukemia cells detected in the meninges of mice 4 weeks after transplantation with dyelabeled (DiR) NALM-6 cells also expressing green fluorescent protein. (J) Mice were transplanted with dye-labeled human NALM- 6 ALL cells ( $2 \times 10^{6} ; n=5$ per group). After 3 weeks, the dye-positive and dye-negative ALL cells were measured and quantified in the meninges and bone marrow by flow cytometry before and after treatment with cytarabine 50 $\mathrm{mg} / \mathrm{kg}$ for 3 days. A relative increase in the dye-positive leukemia cells after cytarabine treatment is consistent with dye-positive leukemia cells being chemoresistant relative to the dye-negative leukemia cells. For all graphs, $* P<0.05, * * P<0.01, * * * P<0.001, * * * * P<0.0001$ by analysis of variance or a $t$-test. 
meninges on leukemia biology. We found that the meninges enhance leukemia resistance to cytarabine and methotrexate, the primary drugs currently used in the treatment of CNS leukemia, by altering the apoptotic balance in leukemia cells to favor survival and increasing leukemia quiescence. ${ }^{1,2}$ Quiescence allows cancer cells to escape cytotoxic chemotherapy and has been shown to be critical for leukemia relapse and stem cell biology. ${ }^{32,51,52}$ In agreement, it was previously shown that high Mer kinaseexpressing, $t(1 ; 19)$ leukemia cells co-cultured with CNSderived cells exhibit G0/G1 cell cycle arrest, suggestive of dormancy or quiescence, as well as methotrexate resistance. $^{16}$

To define the mechanism by which the meninges exert these effects on leukemia biology, we also tested the ability of meningeal conditioned media to enhance leukemia chemoresistance. While meningeal conditioned media partially attenuated the sensitivity of leukemia cells to chemotherapy, the effect was significantly less than when leukemia cells were in direct contact with meningeal cells. This result supports a model in which leukemia chemoresistance is primarily dependent upon direct interactions between the leukemia and meningeal cells with smaller contributions from a soluble factor(s) secreted by the meningeal cells. Together these results further support that the pathophysiology of CNS leukemia and relapse is more complex than simply the ability of leukemia cells or chemotherapy to access the restricted CNS microenvironment and complement other extensive laboratory and clinical data demonstrating that cell-autonomous factors play an essential role in leukemia biology. ${ }^{50,53,54}$

Importantly, we also found that meningeal-mediated leukemia chemoresistance was a reversible phenotype. Leukemia cells removed from co-culture with meningeal cells or the meninges of xenotransplanted mice reverted back to baseline cell cycle, quiescence, apoptosis balance, and sensitivity to methotrexate and cytarabine. Ebinger et al. recently identified a similar population of relapseinducing ALL cells within the bone marrow that exhibited dormancy, stemness, and treatment resistance. ${ }^{31}$ However, similar to our results, these therapeutically adverse properties were reversed when these leukemia cells were dissociated from the bone marrow microenvironment.

We then identified drugs capable of disrupting leukemia-meningeal adhesion. In addition to identifying several drugs that inhibit canonical cell adhesion targets, we also found that Me6TREN, a novel hematopoietic stem cell mobilizing compound, also disrupted leukemiameningeal adhesion in vitro and in vivo. Moreover, Me6TREN enhanced the efficacy of cytarabine in treating CNS leukemia in xenotransplanted mice. In vivo efficacy against two leukemia cell lines with distinct immunophenotypes ( $\mathrm{T}$ - and B-cell) and a primary B-ALL patientderived xenotransplant support the possibility that drugs, or biologic agents, that target leukemia-niche interactions may exhibit broader specificity than mutation-specific therapeutics that are limited by the genetic heterogeneity of leukemia.

The mechanism by which Me6TREN disrupts the leukemia-meningeal niche is an active area of investigation in our laboratory. The ability of MMP inhibitors to diminish the efficacy of Me6TREN agrees with other work showing that Me6TREN upregulates MMP-9 in the bone marrow niche. ${ }^{38}$ Our gene expression data also showed that Me6TREN significantly downregulated the
A
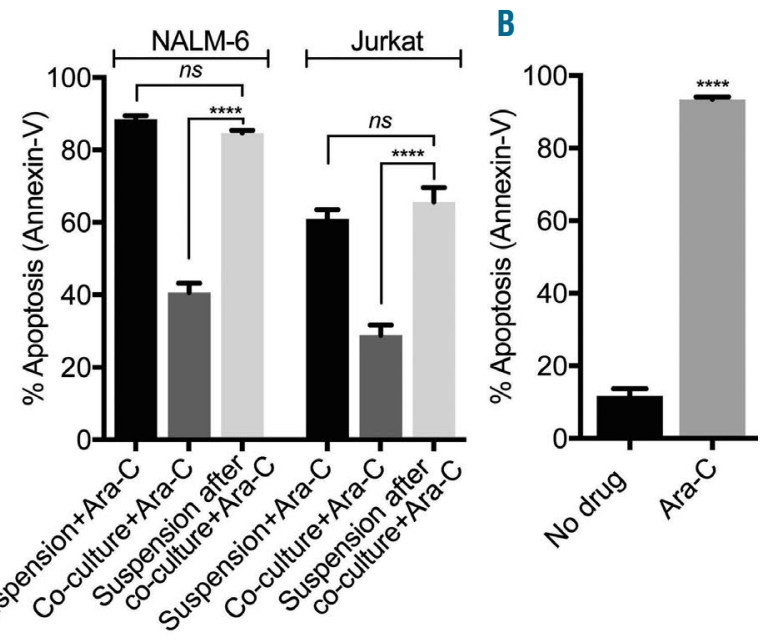

C
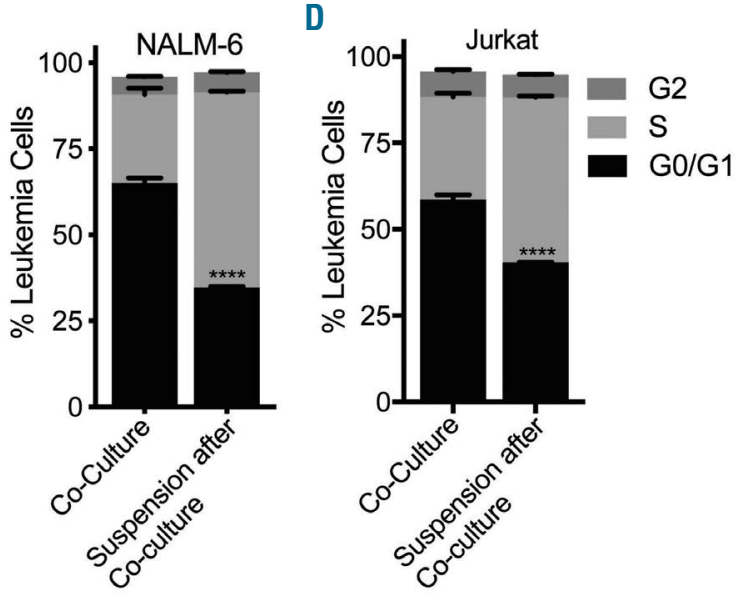

$\mathrm{E}$

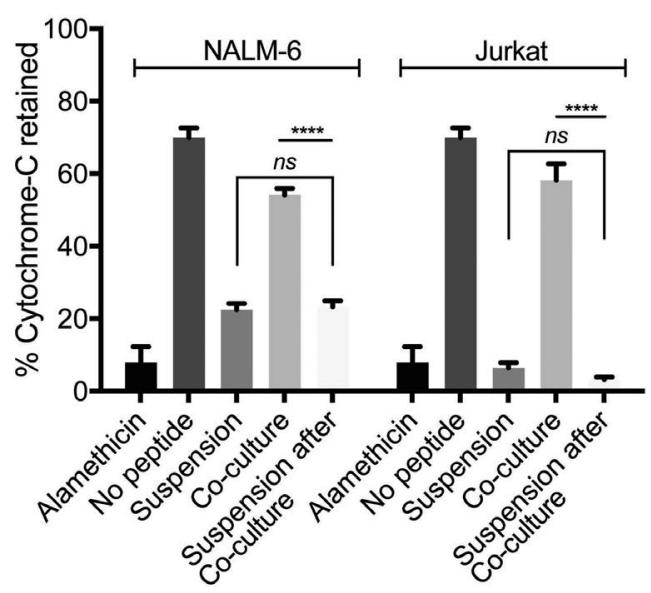

Figure 5. The effects of the meninges on leukemia cells are reversible. (A) NALM- 6 and Jurkat cells grown in suspension, co-cultured with meningeal cells, or isolated from co-culture and placed back in suspension were treated with Ara$C$ (cytarabine) $500 \mathrm{nM}$ and apoptosis was assessed by annexin- $V$ staining and flow cytometry. (B) NALM- 6 leukemia cells were isolated from the meninges of xenotransplanted mice and then grown in suspension for $48 \mathrm{~h}$ prior to treatment with cytarabine $500 \mathrm{nM}$ and assessment of viability using annexin-V staining and flow cytometry. (C, D) NALM-6 (C) and Jurkat (D) leukemia cells were co-cultured with meningeal cells or isolated from co-culture and placed back in suspension and then cell cycle assessed using Click-iT Plus EdU cell cycle reagent and flow cytometry. (E) BH3 profiling was performed using the BIM peptide on NALM- 6 and Jurkat leukemia cells co-cultured with meningeal cells or placed back into suspension after co-culture with meningeal cells. Cytochrome c retention was measured by flow cytometry. For all graphs, data are the mean \pm standard error of mean from three independent experiments. $* * \star * P<0.0001$ by analysis of variance. ns: not significant. 
A

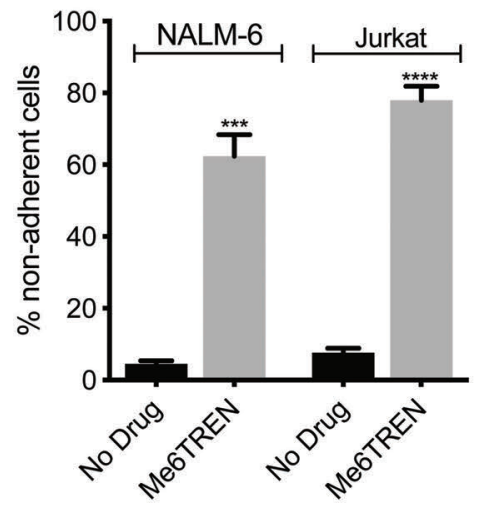

C

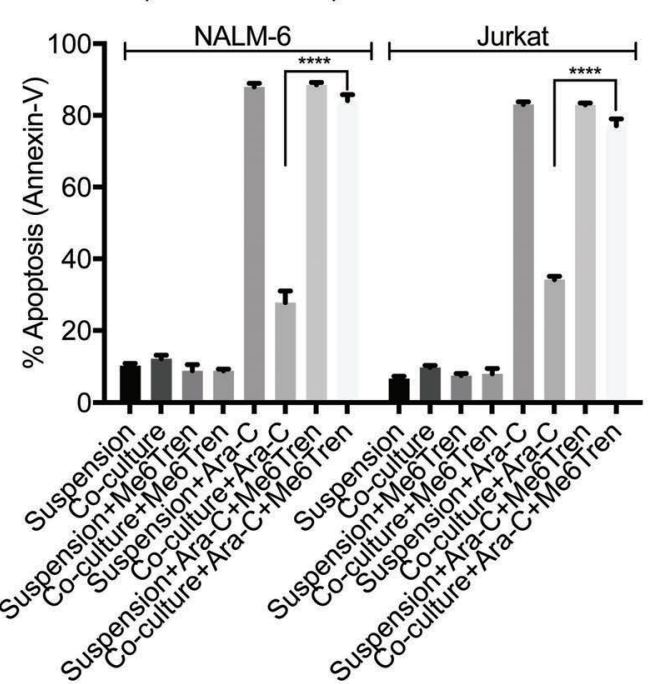

E

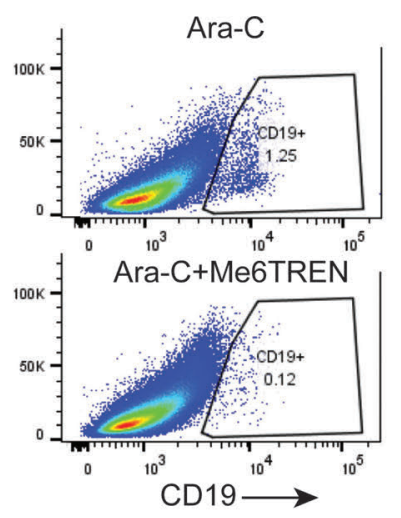

$F$

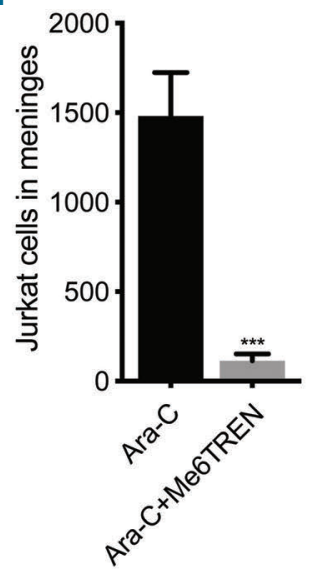

cell-surface/adhesion proteins VCAM-1 and CD99. Supporting a functional role for CD99 and VCAM-1 in leukemia-meningeal adhesion, antibodies targeting either of these proteins attenuated the adhesion of leukemia and meningeal cells. CD99 has not been previously implicated in the mechanism of action of Me6TREN or the ALL niche. Importantly, in addition to directly participating in cell-cell adhesion through homo/heterotypic interactions, CD99 also regulates multiple other adhesion molecules/pathways including LFA-1, $\alpha 4 \beta 1$, ELAM-1, VCAM-1, ICAM-1, MMP, and CXCR4/CXCL12. ${ }^{55}$ As many of these adhesion pathways contribute to the bone marrow leukemia niche, Me6TREN may also diminish
Figure 6. Me6TREN disrupts the meningeal-leukemia niche and attenuates leukemia chemoresistance in vitro and in vivo. (A) NALM-6 leukemia cells were co-cultured with meningeal cells in the presence or absence of Me6TREN $100 \mathrm{uM}$. After $24 \mathrm{~h}$, non-adherent leukemia cells were removed and quantified. (B) Mice were transplanted with NALM-6 leukemia cells $\left(2 \times 10^{6}\right.$ cells; $n=5$ per group). After 16 days, mice were treated with Me6TREN $10 \mathrm{mg} / \mathrm{kg}$ subcutaneous or phosphate-buffered saline control for 3 days. After an additional 24 $\mathrm{h}, 5-10 \mu \mathrm{L}$ of cerebrospinal fluid were removed from the cisterna magna and leukemia cells were quantified using flow cytometry and counting beads. (C) Leukemia cells in suspension or co-cultured with primary meningeal cells were treated with Me6TREN \pm cytarabine 500 $\mathrm{nM}$ and after $48 \mathrm{~h}$ viability was assessed with annexin-V staining and flow cytometry. (D-G) Mice transplanted with NALM-6 ( $2 \times 10^{6}$ cells; $n=5$ per group; $D \& E$ ), Jurkat $\left(2 \times 10^{6}\right.$ cells; $n=5$ per group; $\left.F\right)$, or primary B-cell acute lymphocytic leukemia (PRoXe Sample CBAB-62871-V1; $1 \times 10^{6}$ cells; $\mathrm{n}=5$ per group; $\mathrm{G}$ ) cells were treated with cytarabine $(50 \mathrm{mg} / \mathrm{kg}$ intraperitoneal) or cytarabine + Me6TREN (10 mg/kg subcutaneous). Forty-eight hours after completing therapy mice were euthanized, the heart was perfused, the meninges were isolated, dissociated and stained with human CD19 (NALM-6 \& PDX) or CD3 (Jurkat) antibody, and leukemia cells were quantified by flow cytometry and counting beads $(D, F, G)$. Representative flow plots for NALM-6 are shown in (E). For all graphs, $* P<0.05, \quad * * P<0.01$ $* * * P<0.001, * * * * P<0.0001$ by analysis of variance or a $t$-test.

G

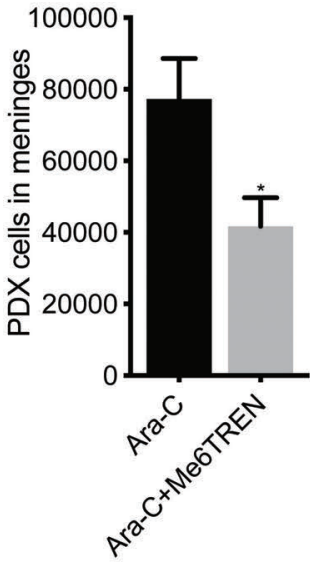

leukemia chemoresistance in other leukemia niches. 53,56 This possibility is potentially supported by our data demonstrating that Me6TREN extended the overall survival of mice treated with cytarabine. However, if upon further testing Me6TREN only disrupts leukemiameningeal adhesion and the effect on survival is due only to decreased CNS leukemia progression, Me6TREN could still be effective in treating isolated CNS relapses, for use in upfront therapy to reduce the risk of CNS relapse, or potentially to reduce the dose or intensity of current CNSdirected therapies that carry significant risks of short- and long-term toxicity.

Given the complexity of cell-cell interactions, this mul- 
tifaceted mechanism of action of Me6TREN may provide an advantage relative to other niche-disrupting agents being developed for leukemia therapy that target a single mechanism of retention or adhesion (i.e. inhibitors of SDF-1 or a single extracellular adhesion factor). ${ }^{57}$ An alternative approach to overcome the complexity and redundancy of cellular adhesion would be to combine multiple inhibitors that target different adhesion molecules (Online Supplementary Figure S6A).

A potential risk of combining Me6TREN, or other niche-disrupting agents, and chemotherapy is that normal hematopoietic stem cells mobilized into the circulation by Me6TREN may be sensitized to chemotherapy with resulting marrow aplasia or delayed hematopoietic recovery. However, we found that mice receiving cytarabine/Me6TREN or cytarabine alone exhibited comparable hematologic toxicities. Moreover, preclinical and clinical studies combining other hematopoietic stem cellmobilizing agents, such as AMD3100 or granulocyte colony-stimulating factor, with chemotherapy have also shown an acceptable toxicity profile. ${ }^{58,59}$ Another potential limitation to this approach of disrupting leukemia cell adhesion to the niche is that soluble factors secreted by the niche can also interact with non-adherent leukemia cells and affect leukemia biology, as we saw with meningeal conditioned media. ${ }^{46,60}$ Combination therapies that target both adhesion and secreted factors may be the most efficacious in treatment of CNS leukemia.

In summary, this work demonstrates that the meninges enhance leukemia chemoresistance in the CNS, elucidates mechanisms of CNS relapse beyond the role of the bloodbrain barrier, and identifies niche disruption as a novel therapeutic approach for enhancing the ability of chemotherapy to eradicate CNS leukemia.

\section{Acknowledgments}

This work was supported in part by the Children's Cancer Research Fund (PMG), the Timothy O'Connell Foundation (PMG), and an American Cancer Society Institutional Research Grant (PMG). PB was partially supported by NIH Training Grant T32 CA099936. We thank Dr. Michael Farrar for providing mouse BCR/ABL p190 leukemia cells, Dr. Juan Abrahante Lloréns (University of Minnesota Genomics Center) for assistance with RNA-sequencing data analyses, Dr. Mark Sanders (University of Minnesota Imaging Center) for providing expert assistance with confocal microscopy and sample preparation, and Mike Ehrhardt (University of Minnesota Cytokine Reference Laboratory) for assistance with measuring cytokine levels. This work utilized the University of Minnesota Masonic Cancer Center shared flow cytometry and comparative pathology resources and the Hematological Malignancy Tissue Bank, which are supported in part by NCI 5P30CA077598-18, Minnesota Masonic Charities, and the Killebrew-Thompson Memorial Fund.

\section{References}

1. Pui C-H, Howard SC. Current management and challenges of malignant disease in the CNS in paediatric leukaemia. Lancet Oncol. 2008:9(3):257-268.

2. Pui C-H, Thiel E. Central nervous system disease in hematologic malignancies: historical perspective and practical applications. Semin Oncol. 2009;36(4 Suppl 2):S2-16.

3. Pui C-H. Central nervous system disease in acute lymphoblastic leukemia: prophylaxis and treatment. Hematology Am Soc Hematol Educ Program. 2006;142-146.

4. Silverman LB. Balancing cure and long-term risks in acute lymphoblastic leukemia. Hematology Am Soc Hematol Educ Program. 2014;2014(1):190-197.

5. Hijiya N, Hudson MM, Lensing S, et al. Cumulative incidence of secondary neoplasms as a first event after childhood acute lymphoblastic leukemia. JAMA. 2007; 297(11):1207-1215.

6. Conklin HM, Krull KR, Reddick WE, Pei D, Cheng C, Pui CH. Cognitive outcomes following contemporary treatment without cranial irradiation for childhood acute lymphoblastic leukemia. J Natl Cancer Inst. 2012;104(18):1386-1395.

7. Krull KR, Brinkman TM, Li C, et al. Neurocognitive outcomes decades after treatment for childhood acute lymphoblastic leukemia: a report from the St Jude lifetime cohort study. J Clin Oncol. 2013;31(35):4407-4415

8. Bhojwani D, Sabin ND, Pei D, et al. Methotrexate-induced neurotoxicity and leukoencephalopathy in childhood acute lymphoblastic leukemia. J Clin Oncol. 2014;32(9):949-959.
9. Iyer NS, Balsamo LM, Bracken MB, KadanLottick NS. Chemotherapy-only treatment effects on long-term neurocognitive functioning in childhood ALL survivors: a review and meta-analysis. Blood. 2015;126(3):346353.

10. Pui C-H, Cheng C, Leung W, et al. Extended follow-up of long-term survivors of childhood acute lymphoblastic leukemia. N Engl J Med. 2003;349(7):640-649.

11. Price RA. Histopathology of CNS leukemia and complications of therapy. Am J Pediatr Hematol Oncol. 1979;1(1):21-30.

12. Price RA, Johnson WW. The central nervous system in childhood leukemia. I. The arachnoid. Cancer. 1973;31(3):520-533.

13. Evans AE, Gilbert ES, Zandstra R. The increasing incidence of central nervous system leukemia in children. (Children's Cancer Study Group A). Cancer. 1970;26(2):404409.

14. Williams MTS, Yousafzai YM, Elder A, et al. The ability to cross the blood-cerebrospinal fluid barrier is a generic property of acute lymphoblastic leukemia blasts. Blood. 2016;127(16):1998-2006.

15. Bartram J, Goulden N, Wright G, et al. High throughput sequencing in acute lymphoblastic leukemia reveals clonal architecture of central nervous system and bone marrow compartments. Haematologica. 2018;103(3):e110-114.

16. Krause S, Pfeiffer C, Strube S, et al. Mer tyrosine kinase promotes the survival of $t(1 ; 19)$ positive acute lymphoblastic leukemia (ALL) in the central nervous system (CNS). Blood. 2015;125(5):820-830.

17. Akers SM, Rellick SL, Fortney JE, Gibson LF. Cellular elements of the subarachnoid space promote ALL survival during chemotherapy. Leuk Res. 2011;35(6):705-711.
18. Gossai NP, Gordon PM. The role of the central nervous system microenvironment in pediatric acute lymphoblastic leukemia Front Pediatr. 2017;5:90.

19. Frishman-Levy L, Izraeli S. Advances in understanding the pathogenesis of CNS acute lymphoblastic leukaemia and potential for therapy. Br J Haematol. 2017;176 (2):157-167.

20. Gaynes JS, Jonart LM, Zamora EA, Naumann JA, Gossai NP, Gordon PM. The central nervous system microenvironment influences the leukemia transcriptome and enhances leukemia chemo-resistance. Haematologica. 2017;102(4):e136-139.

21. Williams RT, Sherr CJ. The ARF tumor suppressor in acute leukemias: insights from mouse models of Bcr-Abl-induced acute lymphoblastic leukemia. Adv Exp Med Biol. 2007;604:107-114.

22. Mishra S, Zhang B, Cunnick JM, Heisterkamp N, Groffen J. Resistance to imatinib of bcr/abl p190 lymphoblastic leukemia cells. Cancer Res. 2006;66 (10):5387-5393.

23. Williams RT, Roussel MF, Sherr CJ. Arf gene loss enhances oncogenicity and limits imatinib response in mouse models of $\mathrm{Bcr}-\mathrm{Abl}$ induced acute lymphoblastic leukemia. Proc Natl Acad Sci USA. 2006;103(17):6688-6693.

24. Townsend EC, Murakami MA, Christodoulou A, et al. The public repository of xenografts enables discovery and randomized phase II-like yrials in mice. Cancer Cell. 2016;29(4):574-586.

25. Liu L, Duff K. A technique for serial collection of cerebrospinal fluid from the cisterna magna in mouse. J Vis Exp. 2008;(21).

26. Manlove LS, Schenkel JM, Manlove KR, et al. Heterologous vaccination and checkpoint blockade synergize to induce antileukemia 
immunity. I Immunol. 2016;196(11):47934804.

27. Montero J, Sarosiek KA, DeAngelo JD, et al. Drug-induced death signaling strategy rapidly predicts cancer response to chemotherapy. Cell. 2015;160(5):977-989.

28. Bhola PD, Mar BG, Lindsley RC, et al. Functionally identifiable apoptosis-insensitive subpopulations determine chemoresistance in acute myeloid leukemia. J Clin Invest. 2016;126(10):3827-3836.

29. Kim KH, Sederstrom JM. Assaying cell cycle status using flow cytometry. Curr Protoc Mol Biol. 2015;111:28.6.1-11.

30. Tario JD, Muirhead KA, Pan D, Munson ME, Wallace PK. Tracking immune cell proliferation and cytotoxic potential using flow cytometry. Methods Mol Biol. 2011;699: 119-164.

31. Ebinger S, Özdemir EZ, Ziegenhain C, et al. Characterization of rare, dormant, and therapy-resistant cells in ccute lymphoblastic leukemia. Cancer Cell. 2016;30(6):849-862.

32. Boyerinas B, Zafrir M, Yesilkanal AE, Price TT, Hyjek EM, Sipkins DA. Adhesion to osteopontin in the bone marrow niche regulates lymphoblastic leukemia cell dormancy. Blood. 2013;121(24):4821-4831.

33. Sipkins DA, Wei X, Wu JW, et al. In vivo imaging of specialized bone marrow endothelial microdomains for tumour engraftment. Nature. 2005;435(7044):969973.

34. Colmone A, Amorim M, Pontier AL, Wang S, Jablonski E, Sipkins DA. Leukemic cells create bone marrow niches that disrupt the behavior of normal hematopoietic progenitor cells. Science. 2008;322(5909):1861-1865.

35. Zuber J, Radtke I, Pardee TS, et al. Mouse models of human AML accurately predict chemotherapy response. Genes Dev. 2009;23(7):877-889.

36. Hiddemann W. Cytosine arabinoside in the treatment of acute myeloid leukemia: the role and place of high-dose regimens. Ann Hematol. 1991;62(4):119-128.

37. Chen $\mathrm{H}$, Wang $\mathrm{S}$, Zhang J, et al. A novel molecule Me6IREN promotes angiogenesis via enhancing endothelial progenitor cell mobilization and recruitment. Sci Rep. 2014;4:6222.

38. Zhang J, Ren X, Shi W, et al Small molecule Me6TREN mobilizes hematopoietic stem/progenitor cells by activating MMP-9 expression and disrupting SDF-1/CXCR4 axis. Blood. 2014;123(3):428-441.

39. Buonamici S, Trimarchi T, Ruocco MG, et al CCR7 signalling as an essential regulator of CNS infiltration in T-cell leukaemia. Nature. 2009;459(7249):1000-1004.

40. Alsadeq A, Fedders H, Vokuhl C, et al. The role of ZAP70 kinase in acute lymphoblastic leukemia infiltration into the central nervous system. Haematologica. 2017;102(2):346355.

41. Münch V, Trentin L, Herzig J, et al. Central nervous system involvement in acute lymphoblastic leukemia is mediated by vascular endothelial growth factor. Blood. 2017;130(5):643-654.

42. Cario G, Izraeli S, Teichert A, et al. High interleukin-15 expression characterizes childhood acute lymphoblastic leukemia with involvement of the CNS. J Clin Oncol. 2007;25(30):4813-4820

43. Wigton EJ, Thompson SB, Long RA, Jacobelli J. Myosin-IIA regulates leukemia engraftment and brain infiltration in a mouse model of acute lymphoblastic leukemia. J Leukoc Biol. 2016;100(1):143153.

44. Holland M, Castro FV, Alexander S, et al. RAC2, AEP, and ICAM1 expression are associated with CNS disease in a mouse model of pre-B childhood acute lymphoblastic leukemia. Blood. 2011;118 (3):638-649

45. Yao H, Price TT, Cantelli G, et al. Leukaemia hijacks a neural mechanism to invade the central nervous system. Nature. 2018;560(7716):55-60.

46. Williams MTS, Yousafzai Y, Cox $C$, et al Interleukin-15 enhances cellular proliferation and upregulates CNS homing molecules in pre-B acute lymphoblastic leukemia. Blood. 2014;123(20):3116-3127.

47. Alsadeq A, Lenk L, Vadakumchery A, et al IL7R is associated with CNS infiltration and relapse in pediatric B-cell precursor acute lymphoblastic leukemia. Blood. 2018;132 (15):1614-1617.

48. Naumann JA, Gordon PM. In vitro model of leukemia cell migration across the bloodcerebrospinal fluid barrier. Leuk Lymphoma. 2017;58(7):1747-1749.

49. Akers SM, O'Leary HA, Minnear FL, et al. VE-cadherin and PECAM-1 enhance ALL migration across brain microvascular endothelial cell monolayers. Exp Hematol. 2010;38(9):733-743
50. Krause DS, Scadden DT. A hostel for the hostile: the bone marrow niche in hematologic neoplasms. Haematologica. 2015;100 (11):1376-1387.

51. Essers MAG, Trumpp A. Targeting leukemic stem cells by breaking their dormancy. Mol Oncol. 2010;4(5):443-450.

52. Norkin M, Uberti JP, Schiffer CA. Very late recurrences of leukemia: why does leukemia awake after many years of dormancy? Leuk Res. 2011;35(2):139-144

53. Chiarini F, Lonetti A, Evangelisti C, et al Advances in understanding the acute lymphoblastic leukemia bone marrow microenvironment: From biology to therapeutic targeting. Biochim Biophys Acta. 2016;1863(3): 449-463.

54. Moses BS, Slone WL, Thomas P, et al. Bone marrow microenvironment modulation of acute lymphoblastic leukemia phenotype. Exp Hematol. 2016;44(1):50-9.e1.

55. Pasello M, Manara MC, Scotlandi K. CD99 at the crossroads of physiology and pathology. J Cell Commun Signal. 2018;12(1):55 68.

56. Gaudichon J, Jakobczyk H, Debaize L, et al Mechanisms of extramedullary relapse in acute lymphoblastic leukemia: reconciling biological concepts and clinical issues. Blood Rev. 2019;36:40-56.

57. Sison EAR, Magoon D, Li L, et al. Plerixafor as a chemosensitizing agent in pediatric acute lymphoblastic leukemia: efficacy and potential mechanisms of resistance to CXCR4 inhibition. Oncotarget. 2014;5(19): 8947-8958.

58. Uy GL, Rettig MP, Stone RM, et al. A phase $1 / 2$ study of chemosensitization with plerixafor plus G-CSF in relapsed or refractory acute myeloid leukemia. Blood Cancer J. 2017;7(3):e542-e542.

59. Cooper TM, Sison EAR, Baker SD, et al. A phase 1 study of the CXCR4 antagonist plerixafor in combination with high-dose cytarabine and etoposide in children with relapsed or refractory acute leukemias or myelodysplastic syndrome: a Pediatric Oncology Experimental Therapeutics Investigators' Consortium study (POE 1003). Pediatr Blood Cancer. 2017;64(8).

60. Laurence ADJ. Location, movement and survival: the role of chemokines in haematopoiesis and malignancy. $\mathrm{Br} \mathrm{J}$ Haematol. 2006;132(3):255-267. 